\title{
Siglec-8 antibody reduces eosinophils and mast cells in a transgenic mouse model of eosinophilic gastroenteritis
}

\author{
Bradford A. Youngblood, ${ }^{1}$ Emily C. Brock, ${ }^{1}$ John Leung, ${ }^{1}$ Rustom Falahati, ${ }^{1}$ Bruce S. Bochner, ${ }^{2}$ \\ Henrik S. Rasmussen, ${ }^{1}$ Kathryn Peterson, ${ }^{3}$ Christopher Bebbington, ${ }^{1}$ and Nenad Tomasevic ${ }^{1}$ \\ ${ }^{1}$ Allakos, Inc., Redwood City, California, USA. ²Division of Allergy and Immunology, Department of Medicine, Northwestern \\ University Feinberg School of Medicine, Chicago, Illinois, USA. 'Division of Gastroenterology, Department of Medicine, \\ University of Utah Health Sciences Center, Salt Lake City, Utah, USA.
}

Conflict of interest: Research was funded by Alkalos, Inc. BAY, ECB, $J \mathrm{~L}, \mathrm{RF}, \mathrm{HSR}, \mathrm{BSB}, \mathrm{CB}$, and NT are or were employees of and/or own stock and/or stock options from Allakos, Inc. BSB is a paid consultant on the scientific advisory board of Allakos, Inc., and owns stock in Allakos. He is a coinventor on an existing Siglec-8related patent (US20080213212A1) and thus may be entitled to a share of royalties received by Johns Hopkins University on the potential sales of such products. He is also a cofounder of Allakos, which makes him subject to certain restrictions under university policy. The terms of this arrangement are being managed by the Johns Hopkins University and Northwestern University in accordance with their conflict of interest policies.

Copyright: (c) 2019, American Society for Clinical Investigation.

Submitted: November 15, 2018

Accepted: August 23, 2019

Published: October 3, 2019

Reference information: JCI Insight. 2019;4(19):e126219.

https://doi.org/10.1172/jci.

insight.126219.

\begin{abstract}
Aberrant accumulation and activation of eosinophils and potentially mast cells (MCs) contribute to the pathogenesis of eosinophilic gastrointestinal diseases (EGIDs), including eosinophilic esophagitis (EoE), gastritis (EC), and gastroenteritis (ECE). Current treatment options, such as diet restriction and corticosteroids, have limited efficacy and are often inappropriate for chronic use. One promising new approach is to deplete eosinophils and inhibit MCs with a monoclonal antibody (mAb) against sialic acid-binding immunoglobulin-like lectin 8 (Siglec-8), an inhibitory receptor selectively expressed on MCs and eosinophils. Here, we characterize MCs and eosinophils from human EG and EoE biopsies using flow cytometry and evaluate the effects of an anti-Siglec-8 mAb using a potentially novel Siglec-8-transgenic mouse model in which EC/EGE was induced by ovalbumin sensitization and intragastric challenge. MCs and eosinophils were significantly increased and activated in human EG and EoE biopsies compared with healthy controls. Similar observations were made in EG/EGE mice. In Siglec-8-transgenic mice, anti-Siglec-8 mAb administration significantly reduced eosinophils and MCs in the stomach, small intestine, and mesenteric lymph nodes and decreased levels of inflammatory mediators. In summary, these findings suggest a role for both MCs and eosinophils in EGID pathogenesis and support the evaluation of anti-Siglec- 8 as a therapeutic approach that targets both eosinophils and MCs.
\end{abstract}

\section{Introduction}

Eosinophilic gastritis (EG) and gastroenteritis (EGE) are rare diseases of the gastrointestinal (GI) tract, affecting minimally 20 in 100,000 people worldwide (1). Prevalence has likely been underestimated because of the absence of a standard diagnosis and medical education $(2,3)$ but is reported to be highest in children and adults ages $20-50$ years, with a slightly higher incidence in men (1). These conditions belong to a larger spectrum of GI disorders known as eosinophilic gastrointestinal disorders (EGIDs), which are characterized by eosinophilic infiltration of the GI tract and debilitating GI symptoms (i.e., abdominal pain, nausea, vomiting, diarrhea, and dysphagia) (1). Current EGID treatment options, such as diet restriction and corticosteroids, have limited efficacy and are often inappropriate for chronic use. Thus, there is a significant medical unmet need for novel targeted therapies.

In EG/EGE and other EGIDs, eosinophils are believed to be one of the principal effector cells responsible for GI symptoms and disease pathogenesis (1). Although mast cells have been reported to be elevated in eosinophilic esophagitis (EoE), their role in EG/EGE is less well understood, despite evidence that EGIDs are atopic in nature (4-6). Development of EG and EGE is associated with a history of allergy, food sensitivity, eczema, and seasonal asthma, implicating involvement of mast cells (1). Furthermore, mast cells and genes associated with the mast cell transcriptome are increased in EG patient tissue (7). Despite elevated numbers in EoE and EG, no further characterization of the role of the mast cell has been performed in EGIDs.

Sialic acid-binding immunoglobulin-like lectin 8 (Siglec-8) is a cell surface receptor that has emerged as a promising therapeutic target for treatment of allergic and inflammatory diseases. Siglec- 8 is an inhibitory receptor that is found selectively on human eosinophils and mast cells (and to a lesser degree, on basophils). Binding of a monoclonal antibody $(\mathrm{mAb})$ to Siglec-8 induces apoptosis to activated eosinophils and 
inhibits mast cell activation (8-10). Only 1 Siglec-8-targeted therapy, AK002, is currently in clinical development for the treatment of allergic, inflammatory, and proliferative diseases involving eosinophils and mast cells. AK002 is a humanized, nonfucosylated IgG1 anti-Siglec- $8 \mathrm{mAb}$ that has been shown to deplete eosinophils via antibody-dependent cellular cytotoxicity and apoptosis and inhibit mast cell function (8).

Given the putative roles of eosinophils and mast cells in EGIDs, we sought to characterize these cell types in EG patient tissue and to develop a mouse model of EG and EGE wherein the effect of an antiSiglec- $8 \mathrm{mAb}$ could be evaluated. Here we show that mast cells and eosinophils are significantly increased and activated in EG and EoE patient tissue biopsies compared with nondiseased tissue. Moreover, the increase in mast cell numbers in these EGID biopsies was similar in degree to that of eosinophils, suggesting that both of these cell types are involved in the pathology of EGIDs. To evaluate the potential activity of an anti-Siglec- $8 \mathrm{mAb}$ in a mouse model of EG and EGE, we developed a potentially novel transgenic mouse that selectively expresses human Siglec- 8 on mast cells, eosinophils, and basophils, as is the case in humans (11). Systemic sensitization and intragastric challenge with ovalbumin (OVA) induced significant infiltration of eosinophils and mast cells in the stomach, small intestine, and mesenteric lymph nodes (MLNs), similar to human EG and EGE disease. Therapeutic administration of an anti-Siglec-8 mAb significantly and selectively reduced eosinophils, mast cells, and inflammation in all these GI tissues. These data provide evidence that both mast cells and eosinophils contribute to EGID pathogenesis and demonstrate that targeting of Siglec-8 suppresses eosinophil and mast cell involvement in an EG and EGE mouse model, thus supporting further clinical investigation of this receptor as a therapeutic target in EGIDs.

\section{Results}

EG and EoE tissues from patients have elevated numbers of both mast cells and eosinophils. Of the EGIDs, EoE is the most prevalent and well characterized, whereas much less is known about EG and EGE. To gain greater understanding of the biology driving EG, we procured fresh gastric tissue from patients with EG or nondiseased control subjects without a history of GI disease (Supplemental Table 1; supplemental material available online with this article; https://doi.org/10.1172/jci.insight.126219DS1). Tissues were digested into single-cell suspensions, and immune cells were characterized by flow cytometry (Figure 1A). To confirm that mast cells and eosinophils were correctly identified in our gating strategy, we examined known surface markers for these cells within the individual gating windows. Mast cells $\left(\mathrm{CD} 45^{+} 7 \mathrm{AAD}^{-} \mathrm{SSC}^{\mathrm{hi}} \mathrm{CD} 24^{-}\right.$ CD16-) expressed Siglec- 8 and CD117 (Supplemental Figure $1 \mathrm{~A}$ ), and eosinophils (CD45+7AAD-SSChi ${ }^{-}$ D24 ${ }^{+} \mathrm{CD} 16^{-}$) expressed Siglec-8, CCR3, and CD11b (Supplemental Figure 1A). To further validate the gating strategy, we sorted mast cells and eosinophils from their respective windows, followed by May-Grunwald Giemsa and H\&E staining, respectively. Cells sorted from the mast cell window routinely contained metachromatic granules that were characteristic of mast cells (Supplemental Figure 1B). Similarly, more than $95 \%$ of cells sorted from the eosinophil window displayed a bilobed nucleus and eosin-pink granules that resembled human eosinophils (Supplemental Figure 1B). These data demonstrate that the gating strategy used in Figure 1A correctly identified mast cells and eosinophils in GI tissue.

As expected, EG tissues had significantly increased numbers of eosinophils compared with nondiseased tissue (Figure 1B). In addition, we found that mast cells were increased by approximately 5-fold in EG tissue compared with nondiseased tissue (Figure 1C). These data were confirmed in EG tissue using additional flow cytometry surface markers for mast cells and eosinophils (Supplemental Figure 1, C-E). Interestingly, mast cells were elevated to a similar extent as eosinophils in EG patient tissues $\left(8.9 \%\right.$ vs. $9.3 \%$ of all CD $45^{+}$cells, respectively). In contrast, the percentage of neutrophils and monocytes was reduced in EG tissue compared with nondiseased control tissue, whereas other immune cells, including T cells, remained unchanged (Figure 1D).

To determine whether these observations could be extended to other EGIDs, we processed and characterized esophageal tissue from patients with EoE. Consistent with findings in EG tissue, as well as previously published findings, mast cells and eosinophils were significantly increased to a similar extent compared with nondiseased esophageal tissue (Figure 1, E and F, and refs. 12-16). These data demonstrate that both eosinophils and mast cells are markedly and proportionally elevated in both EG and EoE patient tissue and support our flow cytometry-based approach to quantitatively assess immune cells in fresh human tissue.

Eosinophils and mast cells in EG and EoE tissue are in an activated state. To characterize the state of activation of eosinophils and mast cells from EG tissue, we examined the expression of surface markers associated with activation by flow cytometry (Figure 2, A and B, and Supplemental Figure 2A). As anticipated, Siglec- 8 was selectively expressed on mast cells and eosinophils from both EG and nondiseased stomach 
A
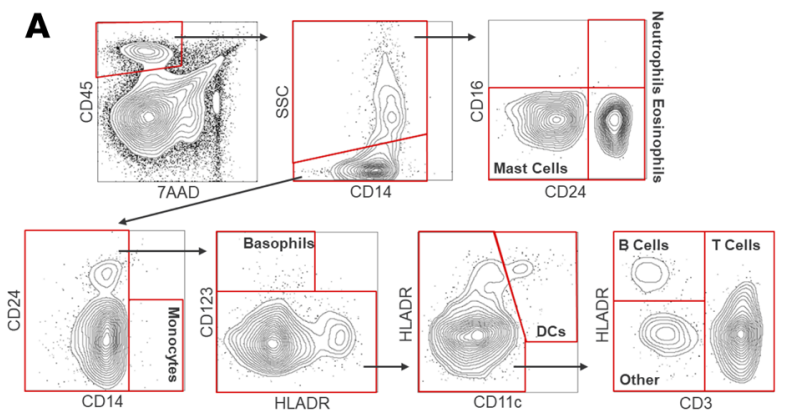

B Stomach Eosinophils

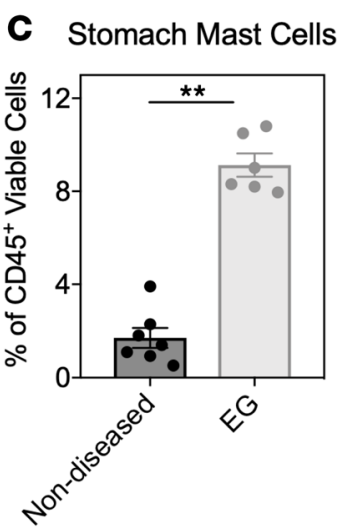

E Esophageal Eosinophils

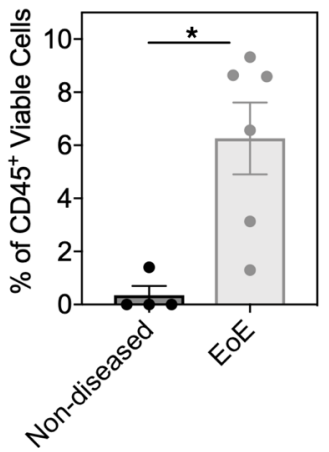

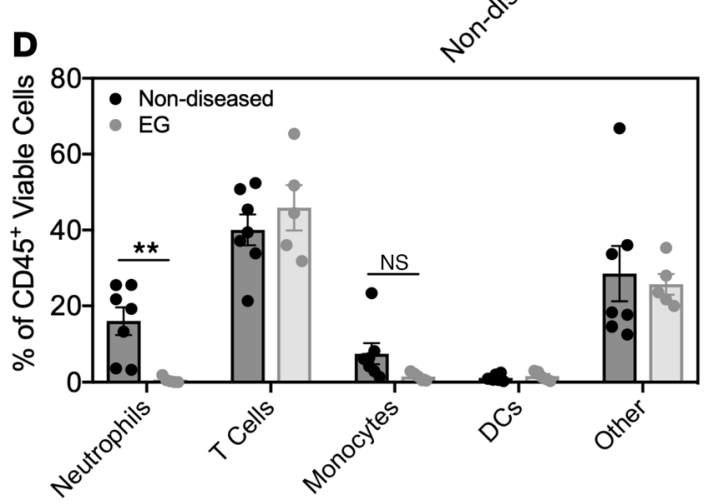

F Esophageal Mast Cells

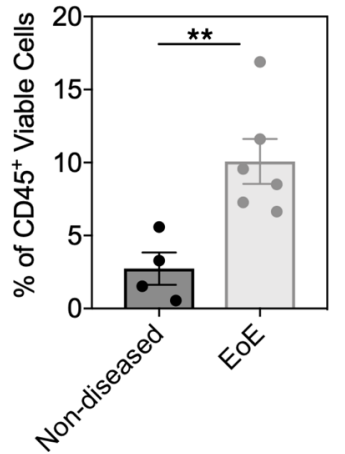

Figure 1. EG and EoE patient tissues have significantly increased numbers of eosinophils and mast cells compared with nondiseased control tissue. (A) Flow cytometry gating strategy used to identify immune cells, including eosinophils and mast cells, in human stomach tissue from patients with EG. Percentage of (B) eosinophils (CD45+7AAD-SS-

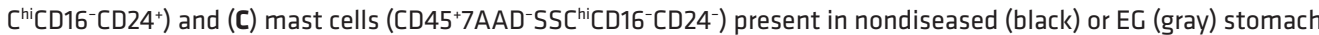
tissue identified using the gating strategy in A. (D) Percentage of neutrophils, T cells, monocytes, DCs, and other ( $B$ cells, NK cells, macrophages, basophils) in nondiseased (black) or EG (gray) stomach tissue using the gating strategy shown in A. Percentage of (E) eosinophils and (F) mast cells present in nondiseased (black) or EoE (gray) esophageal tissue identified using the gating strategy in $\mathbf{A}$. The percentage of cells was derived from the CD45 viable population. Data are plotted as mean \pm SEM for $n=7$ nondiseased stomach tissue and $n=4$ nondiseased esophageal tissue; $n=4$ EG, $n=3 \mathrm{EG}+$ EoE, and $n=3$ EoE patients. ${ }^{*} P<0.05$; ${ }^{*} P<0.01$ by Mann-Whitney $U$ test.

tissues (Figure 2, C and D, and Supplemental Figure 2B). In contrast with Siglec-8, IL-5R $\alpha$ was minimally expressed on EG and nondiseased tissue eosinophils and mast cells (Figure 2C and Supplemental Figure 2C). In addition, tissue eosinophils from patients with EG displayed significantly higher expression of the activation markers, CD11b and CD49d, compared with nondiseased tissue eosinophils, consistent with an increased activation state (Figure 2C and refs. 17-19). Moreover, mast cells from patients with EG displayed significantly increased expression of the degranulation and activation markers CD63 (LAMP3) and CD107a (LAMP1), suggesting an activated and degranulating state (Figure 2D and ref. 20). Consistent with atopy and high serum IgE levels reported for patients with EG (21), EG tissue mast cells also displayed significantly higher levels of surface IgE and Fc\&RI (Figure 2D and Supplemental Figure 2D). 
Last, to determine whether increased mast cell activation occurred in other EGIDs, we examined the same markers on mast cells from EoE patient tissue. Compared with nondiseased esophageal mast cells, EoE mast cells also had significantly higher expression of CD107a, CD63, and IgE (Supplemental Figure $2 \mathrm{E})$. These data demonstrate that eosinophils and mast cells are found in elevated numbers and in an activated state in EG patient tissue. Furthermore, we show that mast cells from EoE patient tissues are also activated and display increased levels of cell surface markers associated with degranulation, such as CD107a. These findings suggest that increased mast cell activation may be a shared feature across EGIDs and support a role for activated eosinophils and mast cells in EGID disease pathogenesis.

Siglec-8-transgenic mice express Siglec-8 on eosinophils, mast cells, and basophils. Based on the data above demonstrating elevation and activation of eosinophils and mast cells in EG patient tissue, we set out to evaluate the activity of an anti-Siglec-8 mAb in a murine disease model of EG and EGE. Like other members of the CD33-related Siglecs, Siglec-8 appears to have evolved recently, with close homologs found only in some primate species $(11,22)$. Therefore, to examine the in vivo activity of anti-Siglec- $8 \mathrm{mAb}$, we generated a human Siglec-8-expressing transgenic mouse, distinct from previously generated mice (e.g., ROSA26-Siglec-8-knockin mice) $(23,24)$. Instead, Siglec-8-transgenic founder mice were generated via the pronuclear microinjection of DNA (Supplemental Figure 3A) as described in Methods. Transmission of the Siglec-8 transgene was successful in 2 of the chimeric founders' progeny (lines 335 and 307), and the transgenic mouse line selected (line 335) contained a single-copy insertion of the Siglec-8 gene (Supplemental Figure 3, B-D). Siglec-8-transgenic mice did not display differences between males or females compared to wild-type (WT) littermates in body weight, weights of major organs, absolute or relative percentages of blood cell types, blood chemistries, or coagulation (Supplemental Figure 3E). Examination of peripheral blood leukocytes (PBLs) and peritoneal lavage (PL) cells from these mice showed high levels of Siglec-8 on the cell surface of mast cells, eosinophils, and basophils in PBLs and PL (Supplemental Figure 4, A-C), consistent with the selective expression of Siglec-8 on human immune cells $(8,11,25)$. Siglec-8 was also found on eosinophils and mast cells in other tissues, including GI, lung, and skin (data not shown), demonstrating expression on both connective tissue and mucosal mast cells. In contrast, Siglec- 8 was not detected on lymphocyte, neutrophil, monocyte, or macrophage cell populations (Supplemental Figure 4, A-C).

To determine whether certain functional properties of Siglec- 8 were preserved in the transgenic mice, such as ligand-induced internalization (26), we engaged the Siglec-8 receptor in vivo by dosing with AK002-G4 (humanized IgG4 [hIgG4] anti-Siglec-8 mAb; ref. 8) or isotype-matched control mAb and examined the extent of receptor internalization. Compared with isotype control mAb-treated mice, antiSiglec- 8 mAb treatment induced Siglec- 8 internalization but not Siglec-F internalization on peripheral blood eosinophils as expected (Supplemental Figure 4, D and E). Finally, insertion of the Siglec- 8 gene in these transgenic mice had no effect on endogenous levels of Siglec-F expression on eosinophils (Supplemental Figure 4E and data not shown).

OVA sensitization and intragastric challenge induces EG and EGE in Siglec-8-transgenic mice. Next, we adapted a previously published experimental study design to create a Siglec-8-expressing murine model of EG and $\operatorname{EGE}(27,28)$. Eosinophils were identified in GI tissue preparations as viable CD $45^{+} \mathrm{Lin}^{-} \mathrm{Ly}^{6} \mathrm{G}^{-} \mathrm{CD}$ $11 \mathrm{~b}^{+} \mathrm{CCR} 3^{+}$Siglec-F $\mathrm{F}^{+}$cells by flow cytometry (Supplemental Figure 5A). GI eosinophils displayed robust Siglec-8 expression, consistent with transgene expression (Supplemental Figure 5A). Systemic sensitization and repeated intragastric challenge with OVA (Figure 3A) induced significant eosinophilic infiltration into the stomach, small intestine, and MLNs compared with sham-treated mice at study takedown on day 39 that resembled EG and EGE (Figure 3, B-G). Consistent with an allergen-specific response, OVA-sensitized and -challenged mice had increased serum levels of OVA-specific IgE and IgG1 compared with sham-treated mice (Figure 3, H and I).

Anti-Siglec- $8 \mathrm{mAb}$ reduces OVA-induced eosinophilic infiltration in the stomach and intestinal tissues. To determine the timing for therapeutic treatment with an anti-Siglec-8 antibody in our EG and EGE mouse model, we examined eosinophils in the periphery, stomach, small intestine, and MLNs after the third OVA challenge on day 32 (Supplemental Figure 6). OVA-sensitized and -challenged mice had significantly increased eosinophils in the stomach, MLNs, and blood compared with sham-treated mice on day 32 (Supplemental Figure 6, A-C). Surprisingly, OVA-exposed mice did not have significantly increased eosinophils in the small intestine at this time point (Supplemental Figure 6D), suggesting the stomach and MLNs are the primary sites of eosinophil infiltration while the small intestine is secondary. Having established EG- and EGE-like disease on day 32 , we selected this time point for therapeutic dosing of the anti-Siglec- $8 \mathrm{mAb}$. 
A
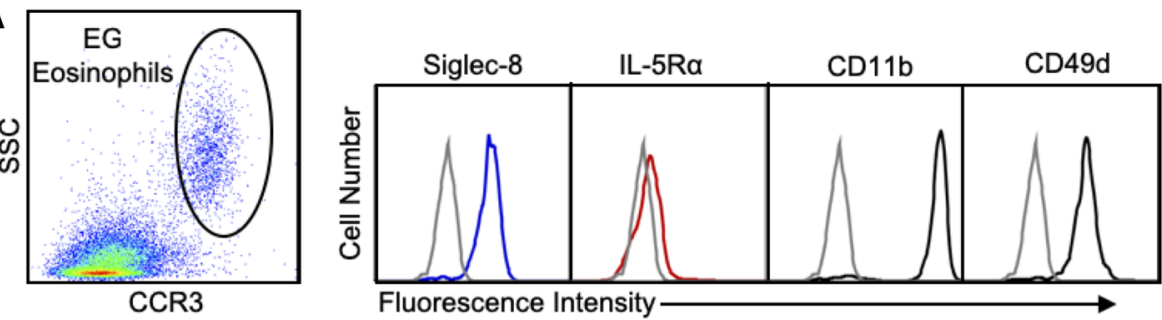

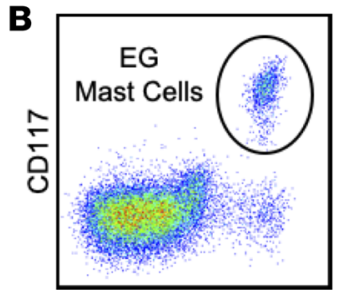

FceRI

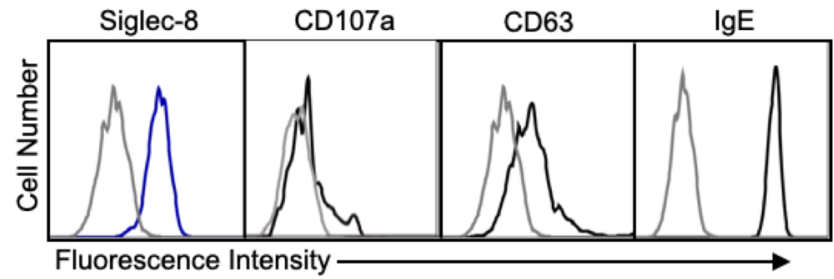

C Stomach Eosinophils
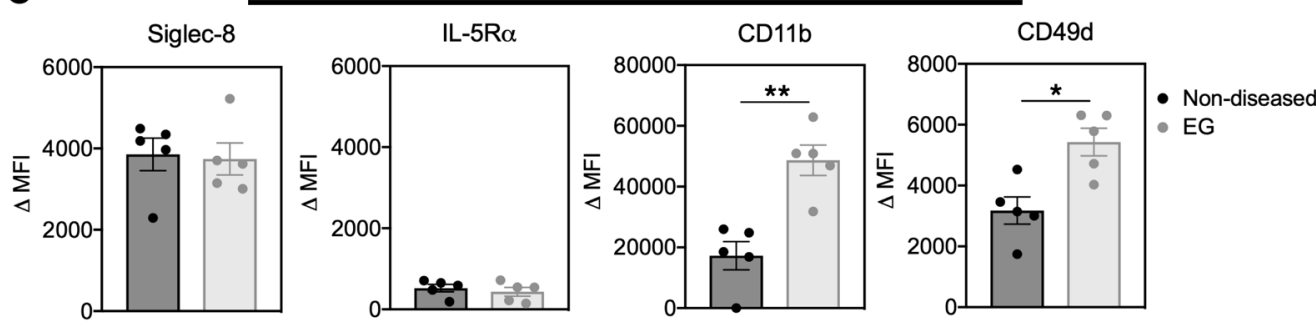

D

Stomach Mast Cells

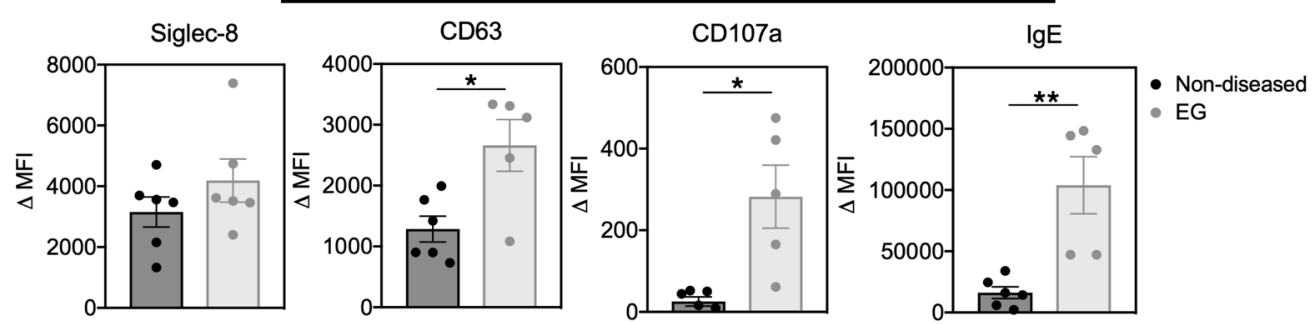

Figure 2. Mast cells and eosinophils from EG and EoE patient tissues are highly activated compared with nondiseased control cells. (A) Dot plot of eosinophils in EG patient tissue identified by CD45+7AAD-CD117-CD16-CCR3 ${ }^{+} S$ SC $^{\text {h }}$ cells. Histogram of EC eosinophils labeled for analysis of surface expression of Siglec-8, IL-5R $\alpha$, CD11b, or CD49d or a fluorescence minus 1 (FMO) negative control (gray). (B) Dot plot of mast cells in EG patient tissue identified by CD45+7AAD-CD117+FcERI+ cells. Histogram of EG mast cells labeled for analysis of surface expression of Siglec-8, CD107a, CD63, or IgE or an FMO negative control (gray). (C) Expression as shown by $\triangle M F I$ of Siglec-8, IL-5R $\alpha$, CD11b, and CD49d on stomach eosinophils from nondiseased controls (black) or patients with EG (gray). (D) Expression as shown by $\triangle \mathrm{MFI}$ of the mast cell activation and degranulation markers, CD63, CD107a, and IgE, on stomach mast cells from nondiseased controls (black) or patients with EG (gray). Data are plotted as mean \pm SD for $n=5-6$ nondiseased stomach tissue; $n=2 \mathrm{EG}$, and $n=3 \mathrm{EG}+\mathrm{EoE} .{ }^{*} P<0.05 ;{ }^{*} P<0.01$ by Mann-Whitney $U$ test.

Therapeutic administration of an anti-Siglec- $8 \mathrm{mAb}$ (mIgG2a) on day 32 led to a significant reduction of eosinophils in the stomach, small intestine, and MLNs at study takedown on day 39, compared with isotype control $\mathrm{mAb}$-treated mice (Figure 4, A-D, and Supplemental Figure 7, A-C). Eosinophil numbers in mice treated with anti-Siglec- $8 \mathrm{mAb}$ were not completely eliminated in the tissue but rather reduced to levels seen in sham-treated mice. In addition to a reduction in tissue eosinophils, mice treated with anti-Siglec-8 mAb had a significant decrease in peripheral blood eosinophils, consistent with the known antibody-dependent cell-mediated cytotoxicity (ADCC) activity of this antibody isotype and subclass (29), compared with sham- and control mAb-treated mice (Figure 4E and Supplemental Figure 7D). To confirm the OVA-induced intestinal eosinophilia seen by flow cytometry, we quantified the mRNA levels of major basic protein (MBP), an eosinophil granule protein, in the small intestine. Mice treated with anti-Siglec-8 
A

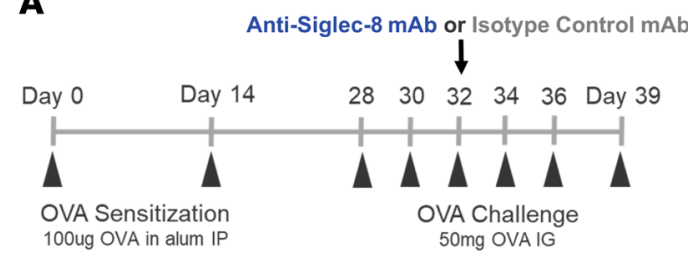

B

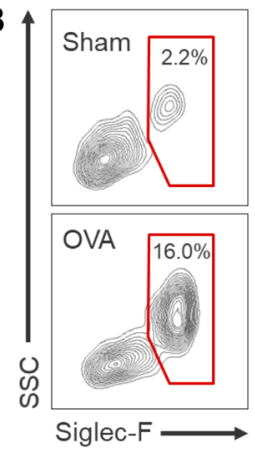

D

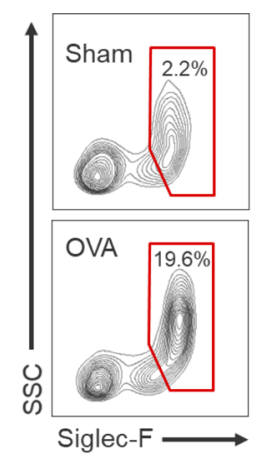

H

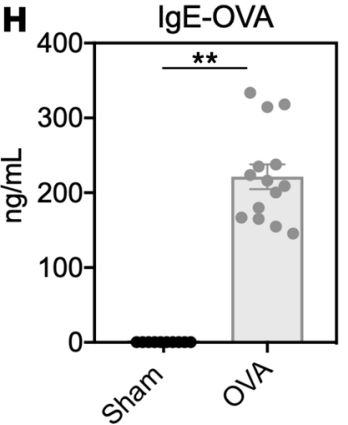

E

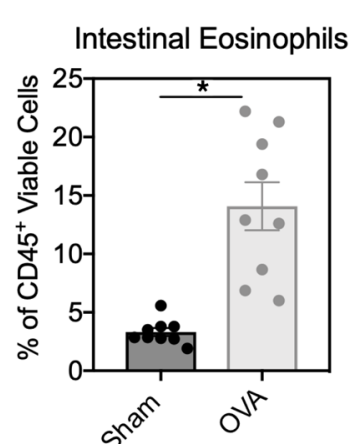

F

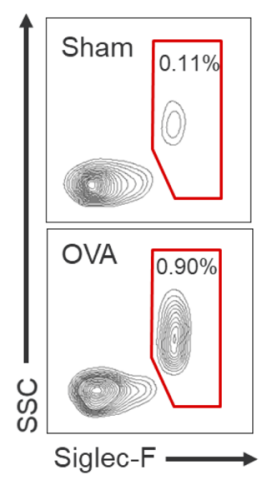

C

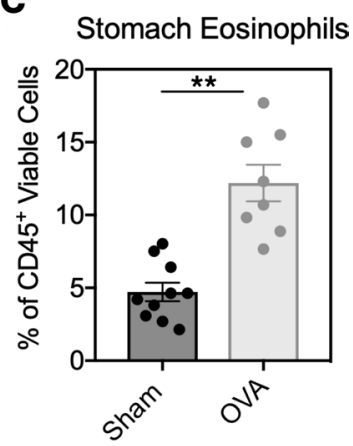

G

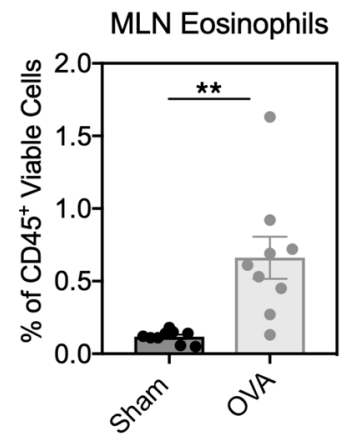

Figure 3. Systemic sensitization and intragastric challenge with OVA induces EG and EGE in Siglec-8-transgenic mice. (A) Schematic of EG and EGE mouse model in Siglec-8-transgenic mice. Mice were systemically sensitized with OVA in aluminum hydroxide adjuvant (alum) on days 0 and 14 , followed by 6 intragastric OVA challenges starting on day 28 until day 39. IP, intraperitoneal. (B) Representative flow cytometry contour plots of stomach eosinophils and (C) the percentage of eosinophils in the stomach in sham- or OVA-administered mice on day 39 quantified by flow cytometry. (D) Representative flow cytometry contour plots of duodenal eosinophils and (E) the percentage of eosinophils in the duodenum in sham-or OVA-administered mice on day 39 quantified by flow cytometry. (F) Representative flow cytometry contour plots of MLN eosinophils and (G) the percentage of eosinophils in the MLNs in sham- or OVA-administered mice on day 39 quantified by flow cytometry. (H and I) Serum levels of OVA-specific IgE or lgG1 in sham-treated mice (black) or mice sensitized and challenged with OVA (gray) on day 39. The percentage of eosinophils is derived from the CD45+ viable cell population. Data are plotted as mean \pm SEM ( $n=8-10$ mice/group) and are representative of 3 experiments. ${ }^{*} P<0.05$; ${ }^{* *} P<0.01$ by Mann-Whitney $U$ test.

$\mathrm{mAb}$ had decreased expression of MBP down to background levels too, similar to the pattern seen with eosinophils in the small intestine compared with control mAb-treated mice (Supplemental Figure 8A). These data demonstrate that anti-Siglec- $8 \mathrm{mAb}$ treatment reduced OVA-induced intestinal tissue eosinophilia in our EG and EGE mouse model.

Anti-Siglec-8 $m A b$ reduces OVA-induced mast cell accumulation in the stomach and intestinal tissues. We also examined mast cell infiltration in the EG and EGE mouse model. Mast cells were identified in GI preparations as viable $\mathrm{CD} 45^{+} \mathrm{Lin}^{-} \mathrm{CD} 117^{+} \mathrm{F} c \varepsilon \mathrm{RI}^{+}$cells by flow cytometry and expressed Siglec-8 (Supplemental Figure $5 \mathrm{~B}$ ). As seen with stomach tissue eosinophils, significantly increased mast cells were found in the stomach but not small intestine by day 32 in OVA-administered mice compared with sham-treated mice (Supplemental Figure 6, E and F). On day 39, significantly increased mast cell numbers were seen in the stomach, small intestine, and MLNs in OVA-sensitized and challenged mice compared with sham-treated mice (Figure 5, A-D; black vs. gray). Therapeutic treatment with an anti-Siglec-8 mAb led to a significant 
A
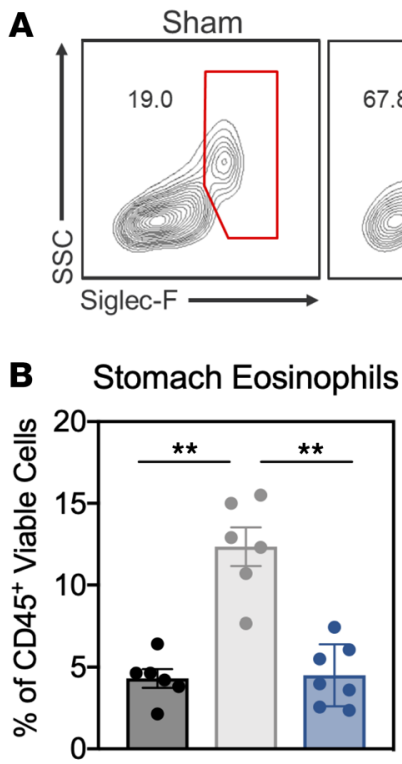

D

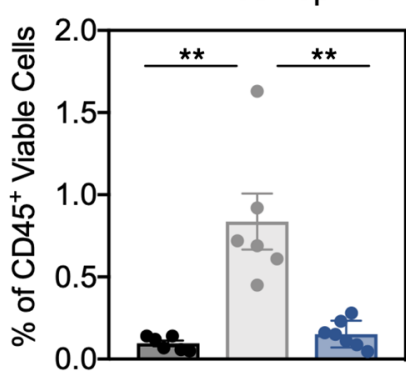

ISO

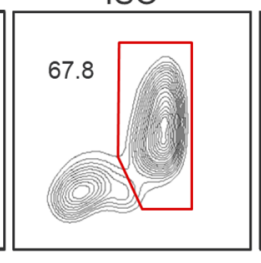

(5)

Gate: Live, Lin

CD45 ${ }^{+}$, Ly6G ${ }^{-}$, CD $11 b^{+}$

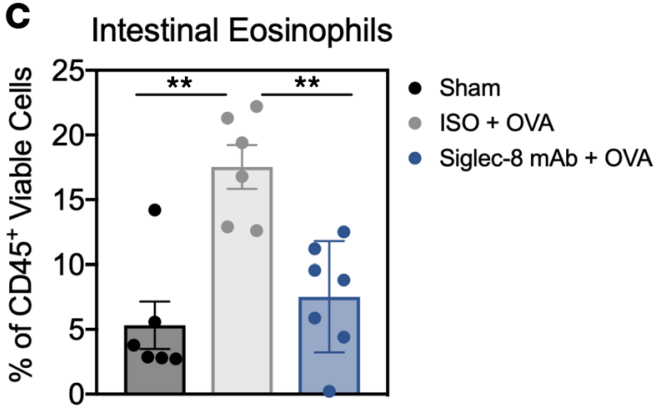

E Blood Eosinophils

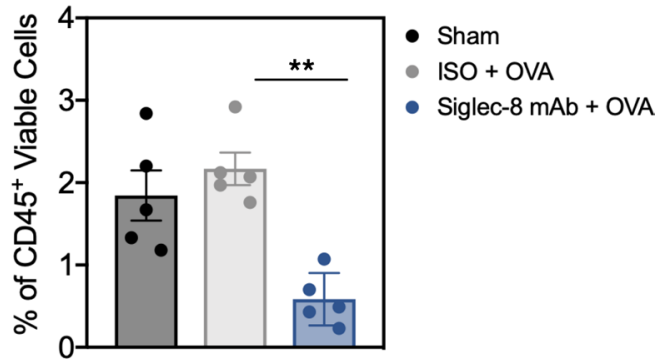

Figure 4. Administration of an anti-Siglec-8 mAb reduces eosinophils in $\mathrm{Gl}$ tissues in mice with EG and EGE. (A) Representative flow cytometry dot plots of stomach tissue eosinophils in mice treated with sham, OVA and isotype control mAb, or OVA and anti-Siglec-8 mAb. The percentage of eosinophils on day 39 in the (B) stomach, (C) duodenum, (D) MLNs, and $(\mathbf{E})$ peripheral blood quantified by flow cytometry in sham-treated mice (black) or mice sensitized and challenged with OVA and dosed with either an isotype control mAb (gray) or anti-Siglec-8 mAb (blue). The percentage of eosinophils is derived from the CD45 viable cell population. Data are plotted as mean \pm SEM ( $n=6-7$ mice/group) and are representative of 3 experiments. ${ }^{* *} P<0.01$ by 1 -way ANOVA with Tukey's multiple-comparisons test.

reduction in the percentage of mast cells in the stomach and small intestine on day 39 compared with control $\mathrm{mAb}$-treated mice (Figure 5, A-C, and Supplemental Figure 7, E and F), albeit not quite back to baseline levels. Similar effects were seen with MLN mast cells but at very low overall cell counts (Figure 5D and Supplemental Figure 7G). To confirm the differences in OVA-induced mast cell infiltration seen by flow cytometry, we quantified the mRNA levels of mast cell protease 1 (MCPT1) in the small intestine. Mice treated with anti-Siglec- $8 \mathrm{mAb}$ had significantly reduced expression of MCPT1 in a pattern similar to changes in mast cell levels, confirming decreased mast cell numbers in the small intestine compared with control mAb-treated mice (Supplemental Figure 8B).

Stomach eosinophils and mast cells are differentially reduced after anti-Siglec- $8 \mathrm{mAb}$ treatment. The cell-specific activity of Siglec-8 on eosinophils and mast cells has been well characterized in vitro and ex vivo (8-10). To evaluate whether the reduction of mast cells and eosinophils in mice treated with anti-Siglec- $8 \mathrm{mAb}$ reflected the known activity of Siglec-8 in vivo, we analyzed stomach tissue on day 32 (before mAb treatment), day 34 (2 days after $\mathrm{mAb}$ treatment), and day 39 in our EG and EGE mouse model. As was seen previously, OVA-challenged mice displayed elevated mast cells and eosinophils in the stomach on day 32 compared with sham-treated mice (Figure 5, E and F). On day 34, 2 days after mAb treatment, mice dosed with an antiSiglec- $8 \mathrm{mAb}$ had significantly decreased eosinophils in the stomach compared with isotype control $\mathrm{mAb}$ treated mice, whereas mast cells decreased only nominally (Figure 5, E and F). On day 39, both stomach eosinophils and mast cells were significantly reduced in mice treated with anti-Siglec- $8 \mathrm{mAb}$; however, the magnitude of decrease seen with eosinophils was greater than that of mast cells (Figure 5, E and F). These data suggest that anti-Siglec- $8 \mathrm{mAb}$ treatment differentially reduces eosinophils and mast cells in the GI tract, consistent with the unique cell-specific activities of Siglec-8 in eosinophils and mast cells.

The faster and more extensive reduction of tissue eosinophils seen in mice treated with anti-Siglec- 8 $\mathrm{mAb}$ compared with mast cells on days 34 and 39 suggest that Siglec- $8 \mathrm{mAb}$ treatment may directly decrease eosinophils in GI tissue. To evaluate this, we collected and cultured dissociated ex vivo stomach tissue from OVA-challenged mice on day 39 overnight in the presence of either an anti-Siglec- $8 \mathrm{mAb}$ or isotype control 
A

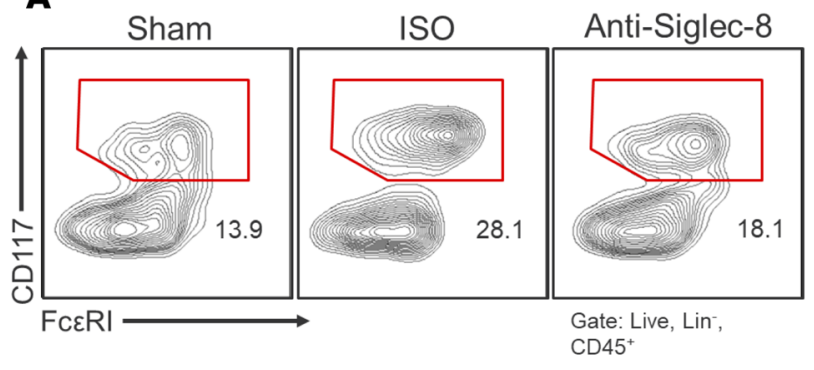

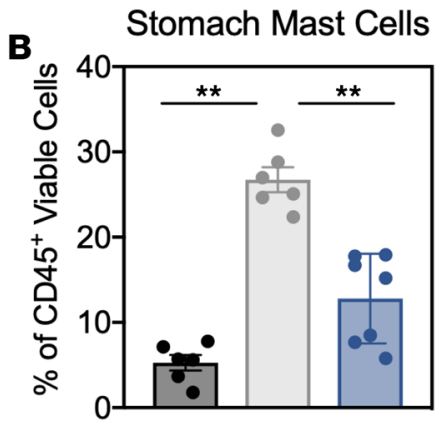

MLN Mast Cells
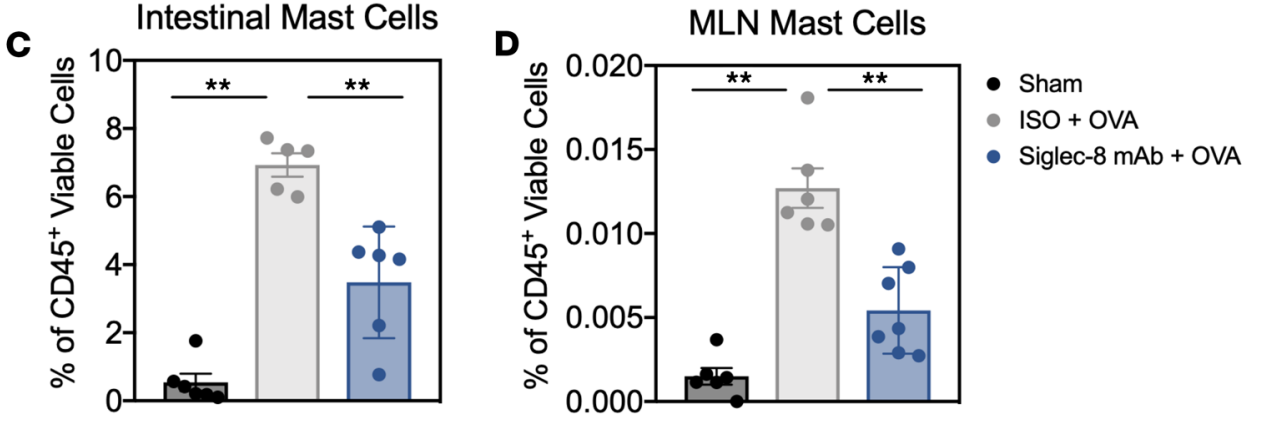

E

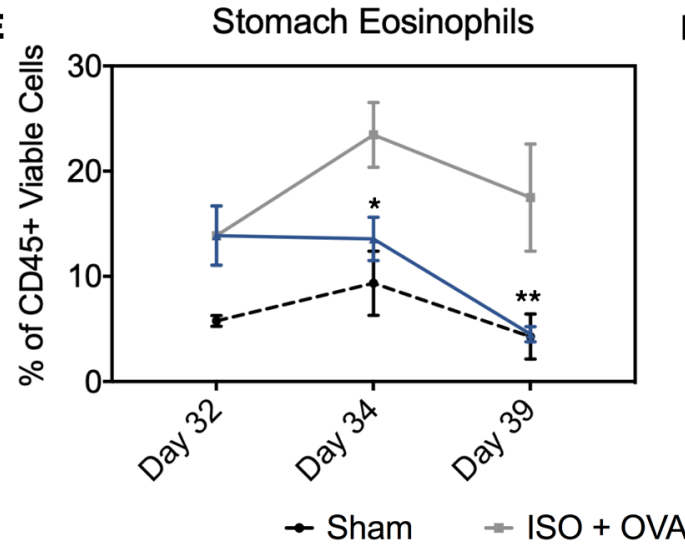

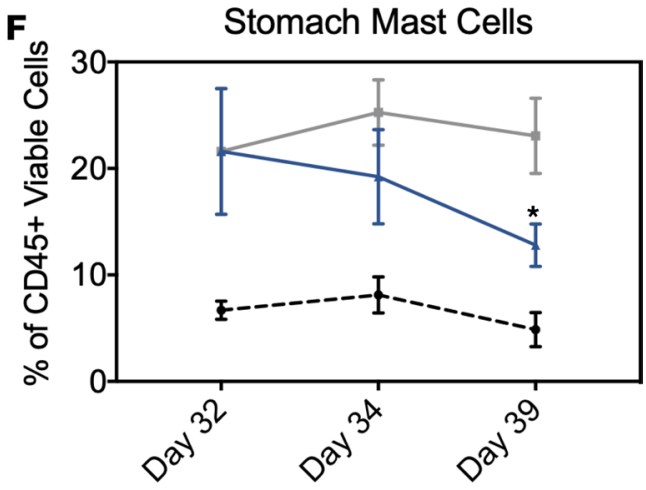

Figure 5. Administration of an anti-Siglec-8 mAb reduces mast cells in Gl tissues in mice with EG and EGE. (A) Representative flow cytometry dot plots of stomach tissue mast cells in mice treated with sham, OVA and isotype control mAb, or OVA and anti-Siglec-8 mAb. The percentage of mast cells on day 39 in the (B) stomach, (C) duodenum, and (D) MLN quantified by flow cytometry in sham-treated mice (black) or mice sensitized and challenged with OVA and dosed with either an isotype control mAb (gray) or anti-Siglec-8 mAb (blue). The percentage of stomach (E) eosinophils or (F) mast cells on days 32,34 , and 39 in mice treated with sham (black), OVA and isotype control mAb (gray), or OVA and anti-Siglec-8 mAb (blue) quantified by flow cytometry. The percentage of mast cells is derived from the CD $45^{+}$viable cell population. Data are plotted as mean \pm SEM ( $n=6-7$ mice/group for B-D and $n=4-6$ mice/group for $E$ and F) and are representative of 3 experiments. ${ }^{*} P<0.05$; ${ }^{* *} P$ $<0.01$ by 1 -way ANOVA with Tukey's multiple-comparisons test (B-D) or 2-tailed $t$ test with Holm-Šídák's posttest (E and F).

$\mathrm{mAb}$, followed by analysis of eosinophils by flow cytometry. Anti-Siglec-8 mAb treatment of dissociated stomach tissue led to significantly fewer eosinophils compared with isotype control mAb-treated tissue (Supplemental Figure 8, C-E). Similarly, AK002 directly reduced human tissue eosinophils in ex vivo lung tissue (8). These data suggest that Siglec- $8 \mathrm{mAb}$ treatment directly reduces GI tissue eosinophils, consistent with the known apoptotic activity of Siglec-8.

Anti-Siglec- $8 \mathrm{mAb}$ reduces OVA-induced inflammation in the intestine and serum. Upon activation with $\mathrm{IgE}$ and allergen, mast cells and subsequently eosinophils elicit inflammatory allergic effects via production of chemokines that drive a type 2 immune response. To evaluate these responses and effects of anti-Siglec- 8 $\mathrm{mAb}$ treatment in the EG and EGE mouse model, we quantified the mRNA expression of known mediators implicated in driving type 2 inflammation in the small intestine. We did not observe increased gene expression of the eosinophil-recruiting chemokine, CCL11, in OVA-challenged mice or detectable expression of the canonical Th2 mediators, IL-4, IL-5, and IL-13, in the intestine (Supplemental Figure 9A). However, the expression of CCL17 (TARC), CCL2 (MCP1), and CCL5 (RANTES) were increased upon OVA challenge in the intestine and decreased with anti-Siglec- $8 \mathrm{mAb}$ treatment compared with isotype control-treated mice (Figure 6, A-C). Furthermore, we measured MLN weight as a surrogate for intestinal inflammation (30). Mice sensitized and challenged with OVA had increased MLN weight compared with sham-treated mice on day 39, and, consistent with the decreased inflammatory signature in the intestine, mice treated with an anti-Siglec- $8 \mathrm{mAb}$ had significantly reduced MLN weights compared with isotype control-treated mice (Supplemental Figure 9B). 
To evaluate systemic changes in mice challenged with intragastric OVA, we examined the expression of cytokines and chemokines in the serum throughout the challenge phase on days 28, 32, 34, and 39. Serum levels of known eosinophil chemokines and cytokines, such as CCL11 and IL-5, were similar in OVA-challenged and sham-treated mice (Supplemental Figure 9, C and D). In contrast, the levels of CCL2, IL-9, and CXCL1 increased throughout the challenge phase in mice exposed to OVA (Figure 6, D and E, and Supplemental Figure 9E). Consistent with the reduction of mast cells and eosinophils in GI tissues, serum levels of CCL2, CXCL1, and IL-9 in OVA-challenged mice on day 39 were significantly reduced with anti-Siglec- $8 \mathrm{mAb}$ therapeutic treatment (Figure 6, F-H). These data demonstrate that the anti-Siglec-8 mAb reduced the expression of several OVA-induced inflammatory mediators associated with eosinophil- and mast cell-driven inflammation.

\section{Discussion}

EGIDs, including EG and EGE, are orphan diseases that are not well managed by current therapies. These studies yielded 2 findings of clinical significance: they provide evidence that in addition to eosinophils, mast cells are elevated in number, express Siglec-8, and display increased levels of degranulation markers within human EG tissue and that a mouse anti-Siglec- $8 \mathrm{mAb}$ can reduce allergen-induced intestinal inflammation in a transgenic mouse model of EG/EGE.

Indeed, because of its selective expression on eosinophils and mast cells, Siglec-8 has emerged as a promising therapeutic target for allergic and inflammatory diseases. Mechanism-of-action studies in humans have demonstrated depletion of blood and tissue eosinophils and inhibition of mast cells with a humanized, nonfucosylated IgG1 anti-Siglec-8 mAb, AK002 (8). Early and ongoing clinical studies with AK002 have yielded promising results, demonstrating rapid depletion of blood eosinophils in healthy volunteers with acceptable safety and tolerability (31). Clinical trials in multiple mast cell- and eosinophil-driven diseases are ongoing.

Although aberrantly high numbers of gastric eosinophils in patients are well documented and serve as the diagnostic criterion for EG and EGE, the involvement of mast cells has not been fully established. A role for mast cells driving EGID pathogenesis is supported by the association of EGIDs with atopic diseases, the effectiveness of dietary therapy, and induction of immediate- and late-phase allergic reactions upon mast cell activation via high-affinity $\operatorname{IgE}$ receptors $(12,13,32)$. Earlier studies in EoE have shown that mast cells are elevated and correlate with clinical symptoms as well as endoscopic and histologic findings (14). To better define the role of mast cells and eosinophils in EG, we used flow cytometry to characterize fresh gastric tissue from EG and nondiseased subjects at the single-cell level and found that both eosinophils and mast cells were selectively and significantly increased in EG gastric tissue. In addition, we found significantly elevated levels of mast cells and eosinophils in tissue from EoE patients, consistent with previously published findings $(15,16,33,34)$. Interestingly, we show that mast cells are elevated by a similar magnitude as eosinophils in both EG and EoE tissue, a finding not previously reported to our knowledge. Surprisingly, we found that the frequencies of neutrophils and monocytes were reduced in EG tissue compared with nondiseased gastric tissue. Although this observation could reflect differences in the type of tissue examined (biopsy vs. resected tissue), it could also be related to the strong Th2 response associated with EG. These hypotheses will be addressed in future studies.

Further evaluation of mast cells and eosinophils in human EG tissue showed that eosinophils from EG tissue were activated, based on the expression of CD11b and CD49d (as previously described with EoE) $(17-19,35)$. Additionally, mast cells from EG and EoE patient tissues displayed significantly increased degranulation markers (CD107a and CD63) and surface-bound IgE, consistent with an atopic state. To our knowledge, this is the first evidence demonstrating that mast cells in EG/EGE tissue are activated and apparently undergoing degranulation, based on changes in surface marker expression. These results suggest that, in addition to eosinophils, mast cells are key effector cells in EGID disease pathogenesis and that therapies that affect only 1 of these cell types may not fully address EGID pathogenesis.

Our analysis confirmed that Siglec-8 was highly expressed on human tissue eosinophils and mast cells, which stands in contrast to the low expression levels of IL-5R $\alpha$. To explore the therapeutic potential of Siglec-8, we used an EG and EGE disease model in Siglec-8-transgenic mice. Development of a transgenic mouse was necessary because of the disparities between human Siglec- 8 and its functional paralog in mice, Siglec-F, most notably, differences in Siglec-8 and Siglec-F function and a lack of expression of Siglec-F on murine mast cells (9). Sensitization and intragastric challenge with OVA led to a significant increase in eosinophils and mast cells in stomach, intestinal, and MLN tissue, correlating with the human ex vivo data described above, further implicating both eosinophils and mast cells in EG pathogenesis. 
A

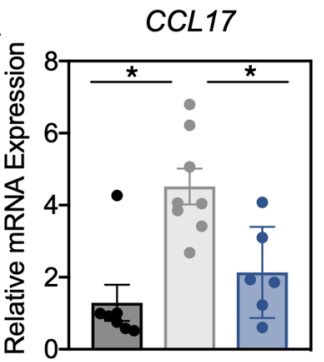

D

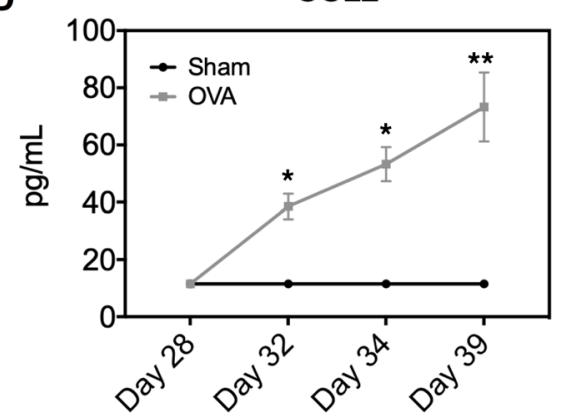

B

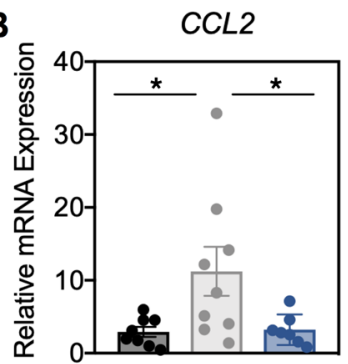

C

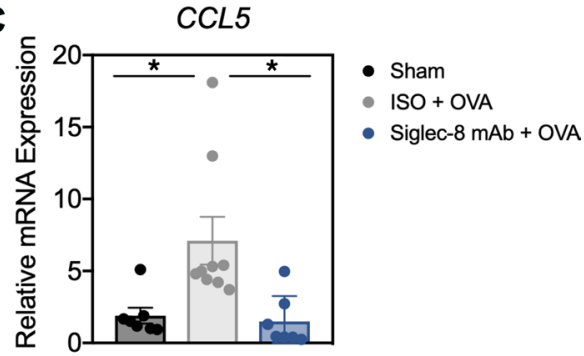

IL-9

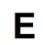

E
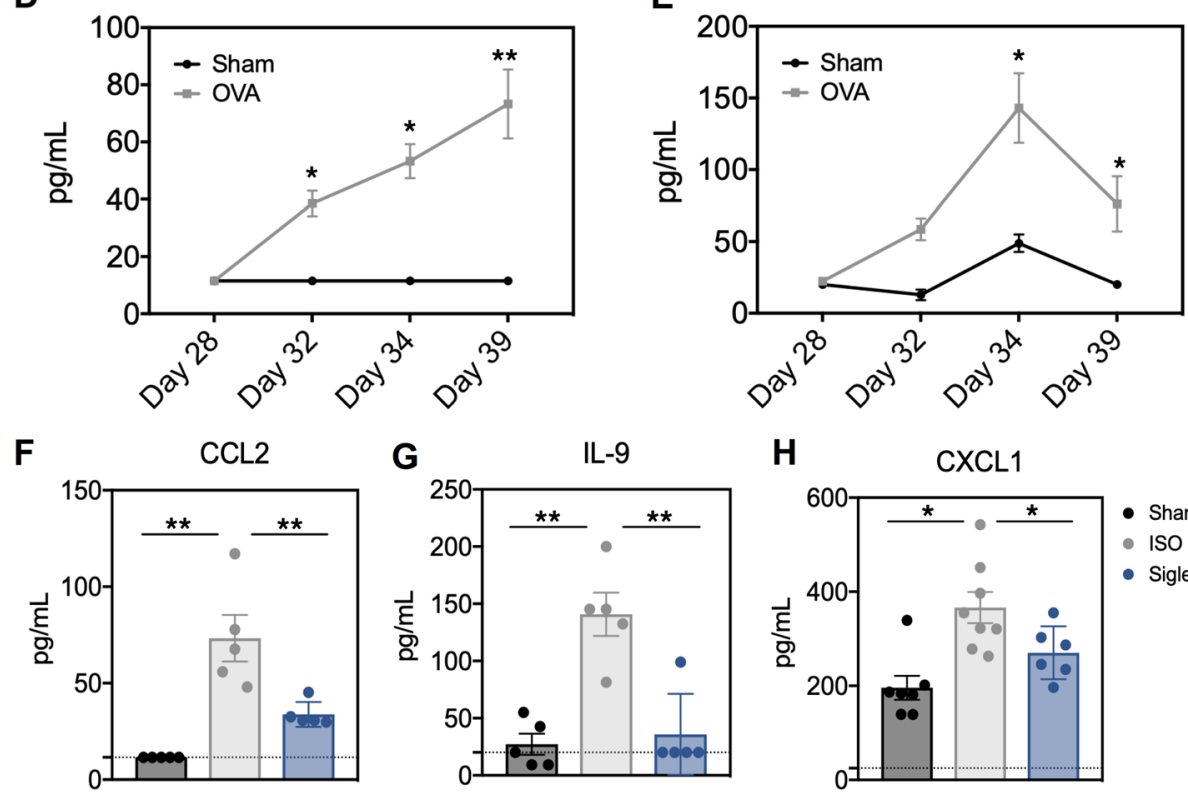

G

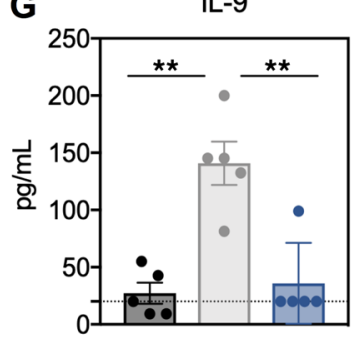

H

CXCL1

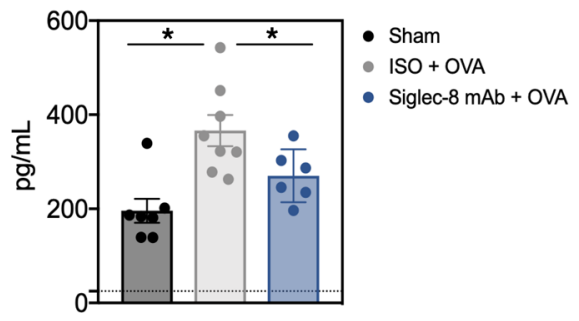

Figure 6. Mice treated with anti-Siglec-8 mAb display reduced expression of OVA-induced type 2 immune-associated inflammatory cytokines and chemokines in intestinal tissue and serum. Quantitative PCR ( $q P C R$ ) gene expression analysis of (A) CCL17, (B) CCL2, and (C) CCL5 in the duodenum at day 39 of study in sham-treated mice (black) or mice sensitized and challenged with OVA and dosed with either an isotype control mAb (gray) or anti-Siglec-8 mAb (blue). ( $\mathbf{D}$ and E) CCL2 and IL-9 levels in serum in sham-treated (black) or OVA-treated (gray) mice on day 28 (before first OVA challenge) and days 32, 34, and 39. (F-H) CCL2, IL-9, and CXCL1 levels in serum in mice treated with sham (black), OVA and isotype control mAb (gray), and OVA and anti-Siglec- $8 \mathrm{mAb}$ (blue) ( $n=5$ mice/group). Graphs are plotted as mean $\pm \operatorname{SEM}\left(n=6-8\right.$ mice/group) and are representative of 3 experiments. ${ }^{*} P<0.05$; ${ }^{*} P<0.01$ by 1 -way ANOVA with Tukey's multiple-comparisons test $(\mathbf{A}-\mathbf{C})$ or 2-tailed $t$ test with Holm-Šídák's posttest ( $\mathbf{D}$ and $\mathbf{E})$.

Treatment with an anti-Siglec-8 antibody reduced mouse eosinophils and mast cells across all GI tissues assessed, as well as decreased blood eosinophils. In this model, GI tissue eosinophils were reduced to sham (baseline) levels in mice treated with anti-Siglec-8 mAb. Interestingly, a similar pattern of eosinophil reduction to sham levels was also seen after anti-Siglec-F antibody treatment in 2 EGID mouse models $(27,36)$. These data suggest, but do not prove, that anti-Siglec-F/8 treatment may preferentially reduce inflammatory eosinophils over the noninflammatory, tissue-resident eosinophil populations in the mouse. Given that the lifespan of murine intestinal eosinophils is estimated to be greater than 7 days $(37,38)$, our data also suggest that anti-Siglec- $8 \mathrm{mAb}$ may elicit direct effects against existing tissue eosinophils, rather than simply on their production. Indeed, ex vivo treatment with an anti-Siglec- $8 \mathrm{mAb}$ significantly reduced murine stomach tissue eosinophils compared with isotype control $\mathrm{mAb}$-treated cells, consistent with our findings of AK002's effect on human tissue eosinophils (8). The reduction of tissue eosinophils by anti-Siglec- $8 \mathrm{mAb}$ or AK002 in ex vivo tissue, where accessory cells are absent, is most likely due to the direct, ADCC-independent induction of death of primed eosinophils. Anti-Siglec- 8 mAb treatment also significantly reduced the number of mast cells in stomach and small intestinal tissue. Because antiSiglec- $8 \mathrm{mAbs}$ are known to have an inhibitory, rather than apoptotic, effect on mast cells, the mechanism of reduction of mature mast cells in the intestine in our model is likely due to the decreased recruitment 
of mast cell progenitors through reduced production of chemokines, including CCL5 and CCL2 $(8,10)$. Indeed, the CCL2/CCR2 axis has been shown to be important in the recruitment of mast cell progenitors in allergic airway inflammation (39), suggesting that CCL2 plays a role in the recruitment of mast cell progenitors to inflamed GI tissue. Alternatively, anti-Siglec- $8 \mathrm{mAb}$ treatment could directly decrease mast cell progenitors or inhibit prosurvival signaling pathways in mature mast cells, resulting in reduced mature mast cell numbers over time. However, Siglec- 8 expression is restricted to mature mast cells, and the turnover of mast cells is thought to be in the range of months, suggesting that mice would need to be dosed with an anti-Siglec- $8 \mathrm{mAb}$ for a longer period to allow for mast cell turnover $(10,40)$. The exact mechanism of Siglec-8-mediated mast cell reduction will be addressed in future studies. Consistent with the apoptotic versus inhibitory activity of Siglec- 8 on eosinophils and mast cells, respectively, we found that eosinophils were reduced faster and to a greater extent than mast cells in mice treated with anti-Siglec- $8 \mathrm{mAb}$. These data may reflect the apoptotic activity of Siglec- 8 on eosinophils and an inhibitory effect on mast cells. In support of Siglec-8-mediated mast cell inhibition in vivo, anti-Siglec-8 mAb treatment has recently been shown to inhibit IgE-mediated passive systemic anaphylaxis in humanized mice (8).

Treatment with the anti-Siglec- $8 \mathrm{mAb}$ also reduced the expression of inflammatory cytokines and chemokines in intestinal tissues and serum. We did not observe increased expression of the canonical Th2 mediators, IL-4, IL-13, and CCL11, despite significant production of OVA-specific IgE and IgG. The lack of change in these Th2-driving cytokines may be attributed to the C57BL/ 6 background of the transgenic mice $(41,42)$. Mice sensitized and challenged with OVA displayed increased expression of other inflammatory cytokines and chemokines associated with type 2 inflammation, including CCL2, CCL5, CCL17, IL-9, and CXCL1, in the intestine and serum. Many of these mediators have been implicated in driving eosinophilic and mast cell infiltration in allergic asthma and food allergy models (43-45). The OVA-mediated increase of cytokines and chemokines was significantly reduced by anti-Siglec- $8 \mathrm{mAb}$, suggesting that mast cells and/or eosinophils may drive their expression. Indeed, CCL2, CCL5, and IL-9 have been shown to be produced by mast cells as well as promote eosinophil recruitment $(43,45-50)$; however, the role of these specific mediators in the stomach and small intestine remains to be elucidated. Consistent with an overall reduction in allergen-induced GI inflammation, mice treated with anti-Siglec- $8 \mathrm{mAb}$ also had decreased MLN weights compared with isotype-treated mice.

In summary, we show that mast cells, as well as eosinophils, are significantly elevated and activated in gastric tissue from patients with EG. Consistent with human disease, our mouse model of EG and EGE also displayed infiltration of eosinophils and mast cells in GI tissues. Therapeutic treatment with an antiSiglec-8 mAb significantly suppressed eosinophil and mast cell accumulation and intestinal inflammation. Collectively, these findings suggest a role for both mast cells and eosinophils in EGID pathogenesis and further support the evaluation of Siglec- 8 as a new therapeutic approach to treat EGIDs.

\section{Methods}

Generation of Siglec-8-transgenic mice. Siglec-8-transgenic founder mice were generated via the pronuclear microinjection of DNA containing the hSiglec- 8 gene and the flanking regions containing the putative native promoter and regulatory elements into $(\mathrm{C} 57 \mathrm{BL} / 6 \mathrm{~J} \times \mathrm{SJL} / \mathrm{J}) \mathrm{F} 2$-fertilized murine eggs and transferred to pseudopregnant recipients using standard methodology at the University of Michigan Transgenic Animal Model Core (51). At 2 weeks of age, viable pups were assayed by PCR for the hSiglec- 8 gene using specific primers (forward primer 5'-AATCAGGTCCCGCCAATAGGAAAAATAATG-3'; reverse primer 5'-CGTGATATAAATCCCCAAGCAACTCCAAT-3'). Transmission of the Siglec-8 transgene was successful in 2 of the chimeric founders' progeny. Siglec-8-transgenic lines 307 and 335 were established and further characterized. Mouse genotypes from tail snips were determined using real-time PCR with specific probes designed for the Siglec- 8 gene by Transnetyx. Raw signal intensity was reported after correcting for the housekeeping gene, c-Jun. ARQ Genetics was contracted to determine the copy number of Siglec-8 in genomic DNA (gDNA) from Siglec-8-transgenic lines compared with normal human gDNA. gDNA was isolated from tail biopsies of transgenic mice, nontransgenic littermate controls, and human blood. Primers within exon 1 of Siglec- 8 were designed using Primer Express 3.0 from Applied Biosystems (forward primer 5'-GGGCCTGTGTGTCCATGTG-3'; reverse primer 5'-CCATGAACTGGGTCAGAGTCAGT-3'). A fluorescein amidite-labeled (FAM-labeled) Siglec-8 probe (5'-CCTGCTCCTTCTCCTACCCCCAGGA-3') was used for qPCR (Applied Biosystems). The amplification curve threshold cycle of the human controls, having a copy number of 2 , was set to a relative quantity (RQ) level of 1.00 ; the mouse line samples were quantified relative to human. 
A mouse line with 1 copy per genome is consistent with an RQ of 0.5 in this assay. The 335 line was selected and backcrossed 6 times to C57BL/6J by Taconic Biosciences under murine pathogen-free health standards before any analysis. Age-matched C57BL/6J WT control mice were purchased from Taconic Laboratories, and Charles River Laboratories was contracted to perform comparisons of age- and sex-matched WT littermates to Siglec-8-transgenic mice. Blood chemistries, coagulation, and hematology were performed by Charles River Research Animal Diagnostic Services (RADS) bioassay services.

Mouse model of OVA-induced EG with gastroenteritis. EGE was induced as previously described (27, 28), in which the induction of EG was a novel finding from the same study design. Briefly, Siglec-8-transgenic mice (8-10 weeks of age) were systemically sensitized with OVA (MilliporeSigma) in $1 \mathrm{mg}$ alum on day 0 and day 14. Beginning on day 28, mice received 6 intragastric OVA challenges every 2 days. Mice received a single intraperitoneal (IP) dose of $5 \mathrm{mg} / \mathrm{kg}$ of either an anti-Siglec- $8 \mathrm{mAb}$ (mIgG2a, Allakos, Inc.) or isotype-matched control mAb (mIgG2a, BioLegend) on day 32. Sham-treated mice were systemically sensitized with OVA and alum but intragastrically challenged with phosphate-buffered saline (PBS; MilliporeSigma). Mice were harvested on day 39 or as otherwise indicated.

Siglec-8 in vivo internalization study. Siglec-8-transgenic mice (8-10 weeks of age) were systemically dosed (IP) with $100 \mu \mathrm{g}$ isotype control (hIgG4, Eureka Therapeutics) or AK002-G4 (hIgG4 anti-Siglec-8 $\mathrm{mAb}$ that does not have ADCC activity) (8). After 48 hours, peripheral blood was collected, and transgenic Siglec-8 and endogenous Siglec-F expression was analyzed on eosinophils using flow cytometry. Conjugated antibodies against non-cross-reactive epitopes were used to detect any remaining Siglec-8 (Allakos, Inc.) or Siglec-F on the cell surface (BioLegend).

Human tissue collection. EG and EoE were diagnosed as at least 30 eosinophils/high-power field (HPF) in $5 \mathrm{HPF}$ in the gastric mucosa and at least 15 eosinophils/HPF in the esophagus without any other cause for the gastric/duodenal eosinophilia (e.g., parasitic or other infection or malignancy). EGID tissue was collected and placed into RPMI 1640 medium (Thermo Fisher Scientific) with penicillin/streptomycin (MilliporeSigma) at the University of Utah and shipped overnight to Allakos, Inc., on wet ice for flow cytometry analysis. Human esophageal and stomach tissue were provided by the National Cancer Institute Cooperative Human Tissue Network from subjects who died from non-GI diseases. Other investigators may have received specimens from the same tissue specimens. All tissues were processed and analyzed approximately 24 hours after collection.

Blood and tissue sample processing. EDTA-treated, anticoagulated blood was collected by cardiac, tail, or submandibular vein bleeding. Peritoneal cells were harvested in PBS. Both were obtained using an IACUC-approved animal protocol. Blood was processed by lysing red blood cells (RBC) with ammonium-chloride-potassium buffer and washed in PBS (Thermo Fisher Scientific) to obtain PBLs. PBLs were resuspended in RPMI 1640 medium and 10\% fetal bovine serum (FBS, Thermo Fisher Scientific). Human and murine intestinal tissue was enzymatically and mechanically dissociated using the gentleMACs Dissociator system (Miltenyi Biotec), according to the manufacturer's protocol. Cells derived from tissue were then treated with RBC lysing buffer, washed in PBS, and resuspended in RPMI 1640 and 10\% low-IgG FBS. Immediately after digestion, cell viability was examined using flow cytometry. From biological fluids and dissociated tissues, only single-cell suspensions that had at least $70 \%$ viability were used in subsequent experiments.

Flow cytometric analysis of blood and tissue. PBLs from murine blood and single cells derived from tissue were obtained as described above. Approximately $1 \times 10^{6}$ to $5 \times 10^{6}$ cells were stained per well in 96-well plates with mouse or human Fc-blocking reagents (BD Biosciences) for 10 minutes at $4^{\circ} \mathrm{C}$ followed by incubation with conjugated antibodies for 10 minutes at $4^{\circ} \mathrm{C}$. Cells were then washed with fluorescence-activated cell sorting buffer $(0.1 \%$ bovine serum albumin in PBS), resuspended in fixative ( $1 \%$ paraformaldehyde in PBS), and analyzed by flow cytometry on a NovoCyte flow cytometer (Acea Biosciences). Antibodies used for identifying immune cell populations in murine tissue included Fc Block (BD Biosciences), CD45 BV785 (BioLegend, 103149), CD3e Biotin (BD, 553060), CD4 Biotin (BD, 553728), CD8a Biotin (BD, 553029), CD19 Biotin (BD, 553784), TER-119 Biotin (BD, 553672), CD5 Biotin (BD, 553019), CCR3 PE (BioLegend, 310714), Ly6G PECy7 (BioLegend, 127618), CD11b APCCy7 (BD, 101257), Siglec-F BV421 (BD, 562681), Streptavadin BV605 (BioLegend, 405229), FceRI PECy7 (BioLegend, 334620), CD49b APCCy7 (BioLegend, 108918), CD117 SB436 (eBioscience, 62117182), and Siglec-8 AF647 (Allakos, Inc.). Live/ dead cells were identified by 7AAD (BD, 559925). Change in MFI was determined by subtracting MFI for an FMO control sample from the MFI for cells stained with a conjugated antibody. Gating strategy for cells from mouse tissues was as follows: mast cells: CD45+Lineage (CD3, CD4, CD8, CD19, CD19, TER-119, 
CD5)-IgER ${ }^{+} \mathrm{CD} 117^{+}$; and eosinophils: CD45 ${ }^{+}$Lineage $-\mathrm{CD} 11 \mathrm{~b}^{+}$Ly6G-Siglec-F $\mathrm{F}^{+} \mathrm{CCR} 3^{+}$. The gating strategy for identifying human immune cells was directly adapted from Yu et al. (52). Antibodies used for identifying immune cell populations in human tissue included CD45 BV785 (BD, 368528), CD14 BV605 (BioLegend, 301834), CD123 BV421 (BioLegend, 563362), CD16 PeCy7 (BD, 557744), CD24 APC Cy7 (BioLegend, 311132), HLA-DR APC (BD, 340549), CD11c PE (BioLegend, 301606), IgER FITC (Miltenyi Biotec, 130095978), CD117 PE/APC (Miltenyi Biotec, 130091733), CD11b PE (BioLegend, 101257), CCR3 PE (BD, 310714), and CD123 BV421 (BD, 563362). Live/dead cells were identified by 7AAD (BD, 559925), IL-5R $\alpha$ PE (BD, 555902), and Siglec-8 PE (R\&D Systems, FAB7975P). Gating strategy for cells from human GI tissue in Figure $1 \mathrm{~A}$ was as follows: mast cells: $\mathrm{CD} 45^{+} \mathrm{SSC}^{\text {hi }} \mathrm{CD} 16^{-} \mathrm{CD} 24^{-}$; eosinophils: $\mathrm{CD}^{2}{ }^{+} \mathrm{SSC}^{\text {hi }} \mathrm{CD} 16^{-} \mathrm{CD} 24^{+}$; neutrophils: $\mathrm{CD} 45^{+} \mathrm{SSC}^{\text {hi }} \mathrm{CD} 16^{+} \mathrm{CD} 24^{+}$; monocytes: $\mathrm{CD} 45^{+} \mathrm{SSC}^{\mathrm{lo}} \mathrm{CD} 14^{+} \mathrm{CD} 24^{-}$; basophils: $\mathrm{CD}^{2} 5^{+} \mathrm{SSC}^{\mathrm{lo}} \mathrm{CD} 14^{-} \mathrm{CD} 123^{+} \mathrm{HLA}_{-\mathrm{DR}}^{-}$; B and T cells: $\mathrm{CD}^{+} 5^{+} \mathrm{SSC}^{\mathrm{lo}} \mathrm{CD} 14^{-} \mathrm{CD} 123^{-} \mathrm{CD} 11 \mathrm{c}^{-} \mathrm{CD} 16^{-}$; and NK cells: $\mathrm{CD}_{45^{+} \mathrm{SSC}}{ }^{\mathrm{lo}} \mathrm{CD} 14^{-} \mathrm{CD} 123^{-} \mathrm{CD} 11 \mathrm{c}^{-} \mathrm{CD} 16^{+}$.

May-Grunwald Giemsa stain. Human GI tissue was processed into single cells as described above. Following digestion, cells were stained with 7AAD, CD45 BV421, CD16 FITC, and CD24 APCCy7 to identify mast cells and eosinophils for flow cytometry. Mast cells (CD45+7AAD ${ }^{-}$SSC $^{\text {hi }}$ CD $16^{-}$CD24and eosinophils $\left(\mathrm{CD}^{4} 5^{+} 7 \mathrm{AAD}^{-} \mathrm{SSC}^{\text {hi }} \mathrm{CD} 16^{-} \mathrm{CD} 24^{+}\right)$were sorted by FACS (Sony Biotechnology) into RPMI 1640 and 10\% FBS (Gibco). Approximately 80,000 mast cells and 100,000 eosinophils were sorted with greater than $95 \%$ purity. Following sorting, cells were centrifuged for 5 minutes at 1200 rpm to create cell pellets and resuspended for cytospin evaluation using a Shandon Cytospin 3 instrument. For May-Grunwald Giemsa staining, the slides were then fixed in methanol for 90 seconds and stained with May-Grunwald Giemsa stain (Newcomer Supply part numbers 1210A and 1121A) according to the manufacturer's instructions. For H\&E staining, the slides were stained with Gill III hematoxylin, transferred to $4.25 \%$ acetic acid, and dipped in eosin. Slides were evaluated by a pathologist, using an Olympus BX51 microscope equipped with a DP72 camera. Representative images were captured using CellSensEntry, v 1.18 (Olympus) at an original magnification of $\times 400$.

Cytokine, chemokine, and OVA-specific IgE and IgG1 analysis in serum and tissue. RNA was isolated from homogenized mouse small intestinal tissue according to methods provided by the manufacturer (Qiagen). cDNA was synthesized according to the manufacturer's protocol (Thermo Fisher Scientific), and qPCR was performed using SYBR green (Thermo Fisher Scientific) and predesigned gene-specific primers for GAPDH, MCPT1, MBP, CCL17, CCL2, CCL5, CCL11, CCL24, IL4, and IL5, and IL13 (IDT). Relative gene expression was calculated using the $\triangle \triangle \mathrm{Ct}$ method. Levels of cytokines and chemokines in mouse serum were quantified using Luminex technology (MilliporeSigma). OVA-specific mouse IgE and IgG was quantified by ELISA according to the manufacturer's procedures (MD Bioproducts).

Statistics. To determine statistical significance, nonparametric Mann-Whitney $U$ test, unpaired 2-tailed $t$ test, 2-tailed $t$ test with Holm-Šídák's posttest, or 1-way ANOVA with Tukey's posttest for multiple comparisons was performed using Prism (GraphPad Software). A $P$ value of 0.05 or less was considered significant.

Study approval. The collection of human EGID biopsy tissue was approved by the University of Utah Institutional Review Board (IRB 00110127). The IRB-approved protocol did not include collection of personal information and medical history from patients besides whether they met diagnostic criteria. All participants provided written informed consent before participating in this study. All animal experiments were done at Aragen Biosciences under an IACUC-approved protocol. For all studies, animals were randomly selected without formal prerandomization, and quantitative measurements were done without the opportunity for bias.

\section{Author contributions}

This work was performed by employees of Allakos, Inc., including BAY, ECB, JL, RF, HSR, BSB, CB, and NT. BAY, ECB, RF, HSR, and NT designed the experiments. BAY, ECB, RF, and JL conducted the experiments. BAY, ECB, and RF acquired data. BAY, ECB, and RF analyzed the data. JL and KP provided reagents. BAY, BSB, ECB, and NT wrote the manuscript.

\section{Acknowledgments}

We thank Robert P. Schleimer for his critical review and feedback on the manuscript, Ingrid Koo and Alan T. Chang for providing writing support during manuscript development, and Ken Luehrsen and Jason Williams for reagent development. 
Address correspondence to: Bradford A. Youngblood, Allakos, Inc., 975 Island Drive, Suite 201, Redwood City, California 94065, USA. Phone: 650.597.5002; Email: byoungblood@allakos.com.

1. Uppal V, Kreiger P, Kutsch E. Eosinophilic gastroenteritis and colitis: a comprehensive review. Clin Rev Allergy Immunol. 2016;50(2):175-188.

2. Bochner BS, et al. Workshop report from the National Institutes of Health Taskforce on the Research Needs of Eosinophil-Associated Diseases (TREAD). J Allergy Clin Immunol. 2012;130(3):587-596

3. Khoury P, et al. Revisiting the NIH Taskforce on the Research needs of Eosinophil-Associated Diseases (RE-TREAD). J Leukoc Biol. 2018;104(1):69-83.

4. Hsu Blatman KS, Gonsalves N, Hirano I, Bryce PJ. Expression of mast cell-associated genes is upregulated in adult eosinophilic esophagitis and responds to steroid or dietary therapy. J Allergy Clin Immunol. 2011;127(5):1307-1308.

5. Abonia JP, et al. Involvement of mast cells in eosinophilic esophagitis. J Allergy Clin Immunol. 2010;126(1):140-149.

6. Kutty GR, Downs-Kelly E, Crispin HT, Peterson KA. Elevated tryptase in EoE is an independent phenomenon associated with extra-esophageal symptoms. Dig Dis Sci. 2019;64(1):152-157.

7. Caldwell JM, et al. Histologic eosinophilic gastritis is a systemic disorder associated with blood and extragastric eosinophilia, TH2 immunity, and a unique gastric transcriptome. J Allergy Clin Immunol. 2014;134(5):1114-1124.

8. Youngblood BA, et al. AK002, a humanized sialic acid-binding immunoglobulin-like lectin-8 antibody that induces antibody-dependent cell-mediated cytotoxicity against human eosinophils and inhibits mast cell-mediated anaphylaxis in mice. Int Arch Allergy Immunol. 2019(2):1-12.

9. Bochner BS. "Siglec"ting the allergic response for therapeutic targeting. Glycobiology. 2016;26(6):546-552.

10. Yokoi $\mathrm{H}$, et al. Inhibition of Fc\&RI-dependent mediator release and calcium flux from human mast cells by sialic acid-binding immunoglobulin-like lectin 8 engagement. J Allergy Clin Immunol. 2008;121(2):499-505.e1.

11. Kikly KK, et al. Identification of SAF-2, a novel siglec expressed on eosinophils, mast cells, and basophils. J Allergy Clin Immunol. 2000;105(6 Pt 1):1093-1100.

12. Gilfillan AM, Austin SJ, Metcalfe DD. Mast cell biology: introduction and overview. Adv Exp Med Biol. 2011;716:2-12.

13. Ko HM, Morotti RA, Yershov O, Chehade M. Eosinophilic gastritis in children: clinicopathological correlation, disease course, and response to therapy. Am J Gastroenterol. 2014;109(8):1277-1285.

14. Tappata M, et al. Association of mast cells with clinical, endoscopic, and histologic findings in adults with eosinophilic esophagitis. Allergy. 2018;73(10):2088-2092.

15. Strasser DS, et al. Eosinophilic oesophagitis: relevance of mast cell infiltration. Histopathology. 2018;73(3):454-463.

16. Spergel JM, et al. New developments in patients with eosinophilic gastrointestinal diseases presented at the CEGIR/ TIGERS Symposium at the 2018 American Academy of Allergy, Asthma \& Immunology Meeting. J Allergy Clin Immunol. 2018;142(1):48-53.

17. Johansson MW. Activation states of blood eosinophils in asthma. Clin Exp Allergy. 2014;44(4):482-498.

18. Bochner BS. Systemic activation of basophils and eosinophils: markers and consequences. J Allergy Clin Immunol. 2000;106(5 suppl):S292-S302.

19. Suzukawa M, et al. Interleukin-33 enhances adhesion, CD11b expression and survival in human eosinophils. Lab Invest. 2008;88(11):1245-1253.

20. Bahri R, et al. Mast cell activation test in the diagnosis of allergic disease and anaphylaxis. J Allergy Clin Immunol. 2018;142(2):485-496.e16.

21. Mori A, Enweluzo C, Grier D, Badireddy M. Eosinophilic gastroenteritis: review of a rare and treatable disease of the gastrointestinal tract. Case Rep Gastroenterol. 2013;7(2):293-298.

22. Hudson SA, et al. Developmental, malignancy-related, and cross-species analysis of eosinophil, mast cell, and basophil siglec-8 expression. J Clin Immunol. 2011;31(6):1045-1053.

23. O'Sullivan JA, et al. Frontline Science: characterization of a novel mouse strain expressing human Siglec-8 only on eosinophils. J Leukoc Biol. 2018;104(1):11-19.

24. Wei Y, et al. Mast cell-specific expression of human Siglec-8 in conditional knock-in mice. Int J Mol Sci. 2018;20(1):E19.

25. Kiwamoto T, Kawasaki N, Paulson JC, Bochner BS. Siglec-8 as a drugable target to treat eosinophil and mast cell-associated conditions. Pharmacol Ther. 2012;135(3):327-336.

26. O'Sullivan JA, Carroll DJ, Cao Y, Salicru AN, Bochner BS. Leveraging Siglec-8 endocytic mechanisms to kill human eosinophils and malignant mast cells. J Allergy Clin Immunol. 2018;141(5):1774-1785.e7.

27. Song DJ, et al. Anti-Siglec-F antibody inhibits oral egg allergen induced intestinal eosinophilic inflammation in a mouse model. Clin Immunol. 2009;131(1):157-169.

28. Brandt EB, et al. Mast cells are required for experimental oral allergen-induced diarrhea. J Clin Invest. 2003;112(11):1666-1677.

29. Overdijk MB, et al. Crosstalk between human IgG isotypes and murine effector cells. J Immunol. 2012;189(7):3430-3438

30. Gao X, et al. Chronic stress promotes colitis by disturbing the gut microbiota and triggering immune system response. Proc Natl Acad Sci U S A. 2018;115(13):E2960-E2969.

31. Rasmussen HS, Chang AT, Tomasevic N, Bebbington C. A randomized, double-blind, placebo-controlled, ascending dose phase 1 study of AK002, a novel Siglec-8 selective monoclonal antibody, in healthy subjects. J Allergy Clin Immunol. 2018;141(2):AB403.

32. Galli SJ, Tsai M. IgE and mast cells in allergic disease. Nat Med. 2012;18(5):693-704.

33. Otani IM, et al. Anti-IL-5 therapy reduces mast cell and IL-9 cell numbers in pediatric patients with eosinophilic esophagitis. $J$ Allergy Clin Immunol. 2013;131(6):1576-1582.

34. Dellon ES, et al. Tryptase staining of mast cells may differentiate eosinophilic esophagitis from gastroesophageal reflux disease. Am J Gastroenterol. 2011;106(2):264-271.

35. Johansson MW, Han ST, Gunderson KA, Busse WW, Jarjour NN, Mosher DF. Platelet activation, P-selectin, and eosinophil 
B1-integrin activation in asthma. Am J Respir Crit Care Med. 2012;185(5):498-507.

36. Rubinstein E, et al. Siglec-F inhibition reduces esophageal eosinophilia and angiogenesis in a mouse model of eosinophilic esophagitis. J Pediatr Gastroenterol Nutr. 2011;53(4):409-416.

37. Jung Y, Rothenberg ME. Roles and regulation of gastrointestinal eosinophils in immunity and disease. J Immunol. 2014;193(3):999-1005.

38. Carlens J, Wahl B, Ballmaier M, Bulfone-Paus S, Förster R, Pabst O. Common gamma-chain-dependent signals confer selective survival of eosinophils in the murine small intestine. J Immunol. 2009;183(9):5600-5607.

39. Collington SJ, Williams TJ, Weller CL. Mechanisms underlying the localisation of mast cells in tissues. Trends Immunol. 2011;32(10):478-485.

40. Bankova LG, Dwyer DF, Liu AY, Austen KF, Gurish MF. Maturation of mast cell progenitors to mucosal mast cells during allergic pulmonary inflammation in mice. Mucosal Immunol. 2015;8(3):596-606.

41. Schulte S, Sukhova GK, Libby P. Genetically programmed biases in Th1 and Th2 immune responses modulate atherogenesis. Am J Pathol. 2008;172(6):1500-1508.

42. Luckett-Chastain L, Calhoun K, Kemp J, Gallucci R. Immunological difference in Th1 and Th2 dominant mouse strains in an ICD model. J Immunol. 2015;194(1 supplement):199.13.

43. Roy RM, Wüthrich M, Klein BS. Chitin elicits CCL2 from airway epithelial cells and induces CCR2-dependent innate allergic inflammation in the lung. J Immunol. 2012;189(5):2545-2552.

44. Kim E, et al. Intestinal epithelial cells regulate gut eotaxin responses and severity of allergy. Front Immunol. $2018 ; 9: 1692$.

45. Lee JB. Regulation of IgE-mediated food allergy by IL-9 producing mucosal mast cells and type 2 innate lymphoid cells. Immune Netw. 2016;16(4):211-218.

46. Toda M, et al. Evidence that formation of vimentin mitogen-activated protein kinase (MAPK) complex mediates mast cell activation following FceRI/CC chemokine receptor 1 cross-talk. J Biol Chem. 2012;287(29):24516-24524.

47. Venkatesha RT, Berla Thangam E, Zaidi AK, Ali H. Distinct regulation of C3a-induced MCP-1/CCL2 and RANTES/CCL5 production in human mast cells by extracellular signal regulated kinase and PI3 kinase. Mol Immunol. 2005;42(5):581-587.

48. Stassen M, et al. IL-9 and IL-13 production by activated mast cells is strongly enhanced in the presence of lipopolysaccharide: NF-kappa B is decisively involved in the expression of IL-9. J Immunol. 2001;166(7):4391-4398.

49. Turner JE, et al. IL-9-mediated survival of type 2 innate lymphoid cells promotes damage control in helminth-induced lung inflammation. J Exp Med. 2013;210(13):2951-2965.

50. Wang A, et al. CCL2/CCR2-dependent recruitment of Th17 cells but not Tc17 cells to the lung in a murine asthma model. Int Arch Allergy Immunol. 2015;166(1):52-62.

51. Van Keuren ML, Gavrilina GB, Filipiak WE, Zeidler MG, Saunders TL. Generating transgenic mice from bacterial artificial chromosomes: transgenesis efficiency, integration and expression outcomes. Transgenic Res. 2009;18(5):769-785.

52. Yu YR, et al. Flow cytometric analysis of myeloid cells in human blood, bronchoalveolar lavage, and lung tissues. Am J Respir Cell Mol Biol. 2016;54(1):13-24. 